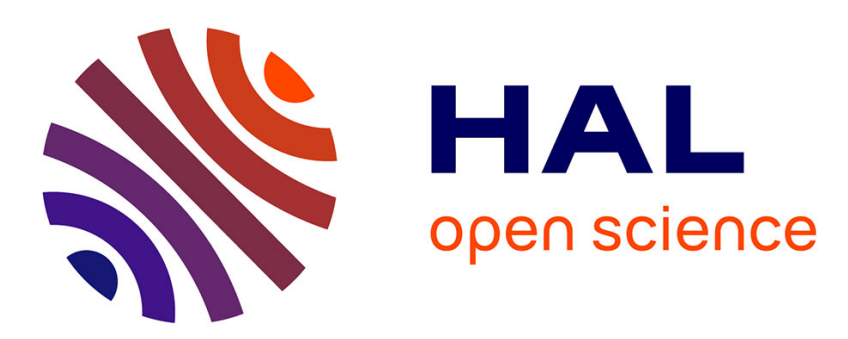

\title{
STUDIES OF LOCAL ORDER IN FLUID STATES BY CROSS CORRELATION INTENSITY FLUCTUATION SPECTROSCOPY
}

N. Clark, B. Ackerson, T. Taylor

\section{- To cite this version:}

N. Clark, B. Ackerson, T. Taylor. STUDIES OF LOCAL ORDER IN FLUID STATES BY CROSS CORRELATION INTENSITY FLUCTUATION SPECTROSCOPY. Journal de Physique Colloques, 1985, 46 (C3), pp.C3-137-C3-147. 10.1051/jphyscol:1985312 . jpa-00224629

\section{HAL Id: jpa-00224629 \\ https://hal.science/jpa-00224629}

Submitted on 1 Jan 1985

HAL is a multi-disciplinary open access archive for the deposit and dissemination of scientific research documents, whether they are published or not. The documents may come from teaching and research institutions in France or abroad, or from public or private research centers.
L'archive ouverte pluridisciplinaire HAL, est destinée au dépôt et à la diffusion de documents scientifiques de niveau recherche, publiés ou non, émanant des établissements d'enseignement et de recherche français ou étrangers, des laboratoires publics ou privés. 


\title{
STUDIES OF LOCAL ORDER IN FLUID STATES BY CROSS CORRELATION INTENSITY FLUCTUATION SPECTROSCOPY
}

\author{
N.A. Clark, B.J. Ackerson ${ }^{\star}$ and T.W. Taylor \\ Department of Physics, University of Colorado, Boulder, Colorado 80309, U.S.A. \\ "Department of Physics, OkZahoma State University, Stillwater, \\ OkZahoma 74078, U.S.A.
}

\begin{abstract}
Résumé.- On prèsente une expression formelle de la fonction de corrêlation de la fluctuation d'intensité (CCIFS) en terme des fonctions de corrélation à $\mathrm{N}$ particules. On étudie cette expression dans plusieurs cas limites et on montre qu'elle est reliée à un type de fonctions de corrélation qui décrivent des expériences actuelles ou futures. La CCIFS entière est nécessaire pour conprendre certaines expëriences récentes mettant en jeu des processus de diffusion dans de petits volumes contenant des particules fortement corrélées. Cependant, la CCIFS entière est compliquée et 1 'on étudie par simulation numérique un système de disques durs afin d'acquêrir quelques aperçus en la matière. On en conclut que les cCIFs doivent en général inclure des corrélations à 3 ou 4 particules pour donner une approximation suffisante de la CCIFS. On discute enfin quelques usages possibles de la technique de la CCIFS.
\end{abstract}

Abstract.- A formal expression is presented in terms of multi-particle correlation functions for the cross-correlation intensity fluctuation (CCIFs) function. This expression is examined in several limits and is seen to be related to a variety of correlation functions which describe present or proposed experiments. Evidently the full CCIFS function is required to understand recent CCIFS experiments for small scattering volumes containing strongly correlated particles. However, the full CCIFS function is rather complex and a two dimensional hard disk system is investigated by computer simulation to gain better insight. It is found that the resulting CCIFS functions must include three and four particle correlation functions, in general, to give accurate representation of the CCIFS correlation function. A model calculation is reviewed and possible uses of the CCIFS technique are discussed.

\section{Introduction}

Scattering techniques have proved to be a fruitful method for investigating the many body order and structure in condensed matter systems. X-ray and elastic neutron scattering are used routinely to orient and to determine the structure of crystalline atomic and molecular solids. ${ }^{1}$ Similarly, protein structures may be determined if these complex molecules can be condensed into a regular crystalline solid. 2 In a like fashion light scattering may be used to determine the structure of "colloidal solids" which are comprised of monodisperse, submicron, plastic, spheres suspended in water and which have lattice constants on the order of the light prope wavelength. 3 In addition to Bragg scattering, thermal diffuse scattering ${ }^{4}$ and Kossel lines ${ }^{5}$ may also be observed by these scattering techniques and yield elastic constant data in addition to structural information. Inelastic neutron, neutron spin-echo and quasi-elastic light scattering techniques can be used to determine dispersion relations and elastic constants in solid atomic and colloidal state samples. 
In liquid samples inelastic techniques may be applied to investigate the hydrodynamic modes (small momentum transfer-long time scale) to determine transport coefficients such as thermal diffusivities, self diffusion coefficients, and viscosity co-efficients. 7,8 The structure in liquid samples may be inferred from the same elastic neutron, $x$-ray and light scattering techniques used to investigate solid systems. The structural information obtained on liquids is characterized by the pair correlation function $g(r)^{8}$, which gives the probability for finding a particle at a distance $r$ given another particle at the origin. In a solid sample the long range order and lack of rotational symmetry permits determination of the crystalline structure from the pair correlation function despite lack of phase information. In the liquid state, however, there is rotational symmetry about any given particle; and as a result, there is much less information in this function. For example, the coordination of nearest neighbors about a given particle is directly observed in scattering from crystalline solids but can only be inferred indirectly for the liquid state. When scattering from a liquid, the spherically symmetric pair distribution function must be found by a fourier inversion of the scattering data. 8 From the area under the first peak in the pair distribution function the number of nearest neighbors is estimated. Coordination of these particles can then be inferred by assuming a local crystalline solid order and using that solid structure which exhibits the same coordination. Indeed many years ago, Prins modelled the liquid structure as a local solid structure where the position of the coordination shell about a given particle becomes more random as the distance of the coordination shell from the particle increases.

Alternate proposals for the existence of local structure in liquids have focused recently on icosohedral order in liquid or glassy states. 10 This icosohedral structure has a higher local packing density than face centered cubic (fcc) or hexagonal close packed (hep) structures. Yet it is only a local structure and cannot be extended indefinitely without introducing defects. However, an amorphous state characterized by these local icosohedral structures may be thermodynamically stable as a result of more efficient local packing. 11 The fact that these icosohedral structures are locally stable may explain the existence of the glassy state. Such structures appear with decreasing temperature until they involve nearly all of the particles. Their local stability frustrates the formation of a periodic solid. Evidence for these structures is sketchy and is difficult to determine unambiguously from standard scattering experiments due to similarities in nearest neighbor coordination numbers with other structures.

Standard scattering experiments are also insensitive to the growth of bond orientational order ${ }^{12}$ in liquid samples as freezing occurs (in 2-dimensions). Furthermore, the large sample volumes used in standard scattering equipment also limit the ability to look at fluid particle order near liquid-solid phase boundaries. Thus there seems to be ample reason to investigate alternative scattering techniques which are tailored to measure the average local order and coordination in fluid states. In the following sections we introduce and discuss such a technique, cross correlation intensity fluctuation spectroscopy ${ }^{13}$, CCIFS. This is a nongaussian scattering technique which employs at least two spatially separated detectors directed at a scattering volume which is comparable in size to the fluid sample correlation length. Local order and coordination may be investigated as the CCIFS function depends on particle distribution functions up to fourth order. In the following section we develop an intuitive basis for cross correlation and experimental results are presented for cross correlation studies of a two dimensional colloidal liquid in the following section. Theoretical developments are presented and followed by a discussion in the final section.

\section{Intuitive Description of Cross Correlation Spectroscopy}

Figure la displays a 2-dimensional liquid structure for a hard disk fluid. As expected in the fluid phase, this sytem possesses no long range transiational or orientational order. However, there is considerable local order. A given particle maintains a fairly well defined distance from its nearest neighbors. 
This coordination of nearest neighbors is generally six (hop) though some five and seven coordination numbers can be seen. Even some square packing is evident.

(a)

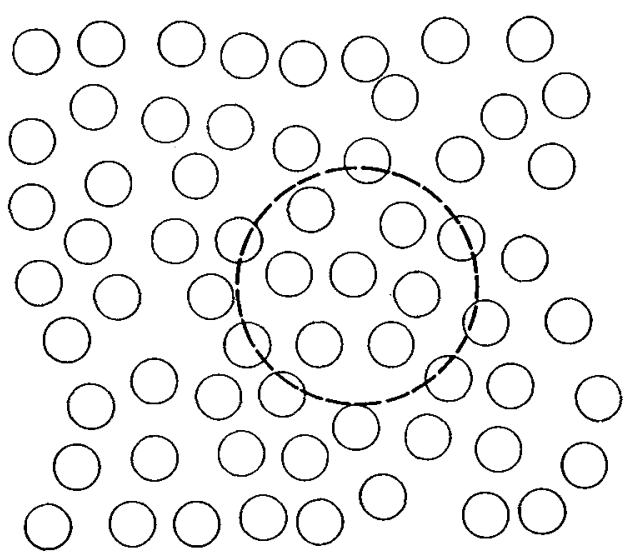

(b)

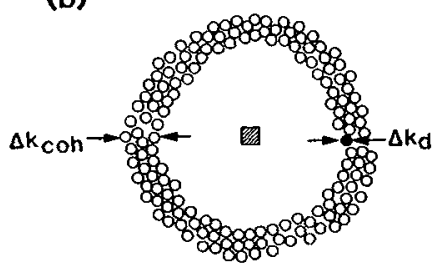

(c)

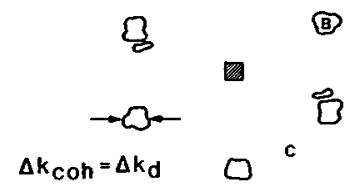

Fig. 1. (a) Monte Carlo simulation configuration for a hard disk fluid with a packing fraction 0.76 . The primary particle coordination number is six (hop structure). However there are five and seven particle coordination numbers visible, as well as, square structures. The dashed circle encloses a single fluid correlation region. (b) Typical Debye Scherrer ring produced by scattering radiation from a large sample of fluid. The ring radius $k_{d s}$ is inversely proportional to the average interparticle separation. The width of the ring $\Delta \mathrm{k}_{\mathrm{coh}}$ is inversely proportional to the fluid coherence area. The speckle size $\Delta \mathrm{k}^{\mathrm{coh}} \mathrm{is}$ inversely proportional to the scattering volume dimension. (c) Scattering pattern produced when the scattering area is on the same order as the fluid correlation area. This pattern would be produced by illuminating the region of fluid enclosed by the dashed line in part (a).

A standard scattering experiment, which illuminates the whole sample shown in figure 1, will produce a typical liquid like scattering pattern, which is azimuthally symmetric about the probe beam. The first Debye Scherrer ring will have a position $k_{D S}$ characteristic of the average local order and a width

$\Delta \bar{k}_{\mathrm{COH}}$ characteristic of the fluid correlation range. The speckle size $k_{d}$ is related to the scattering volume dimension d. The ring is smooth and symmetric because there are many local crystal-like structures of random orientation in the scattering volume. This is similar to the smooth rings produced by scattering from crystalline powders, where the large number of scattering centers insures a Gaussian distribution of the scattered field amplitude.

As the size of the scattering volume is reduced, the number of illuminated "crystallites" is reduced. The scattered field amplitude becomes less Gaussian, and the scattering pattern becomes grainy or speckled in appearance. The scattered beam becomes diffraction broadened, and is equal in width to $\Delta \mathrm{k}_{\mathrm{CH}}$ when the fluid correlation region and scattering volume are comparable. Under these conditions single locally ordered structures are being illuminated. Instantaneous "snap shots" of the scattering pattern (Figure lc and 
figure 2c-f) will reflect this structure showing the symmetry and coordination of the local crystal-like structure. These patterns evolve in time and take on random orientations as the fluid fluctuates in structure. Thus the time averaged scattered intensity will exhibit the same structure as the standard scattering from the liquid phase with the exception that it will be diffraction broadened due to the finite size of the scattering volume. However, if two detectors are employed and cross correlated, then the average local structure can be examined. When the local fluid orientation is such that one detector (labelled $A$ in $f$ igure 1c) is illuminated, counts are then accepted by a second detector. The second detector may be positioned (labelled $B$ in $f$ igure $1 \mathrm{c}$ ) such that it is illuminated at the same time giving a positive cross correlation; or it may be positioned (labelied $C$ in figure 1c) such that it is dark when the other is illuminted, giving no cross correlation signal or an anticorrelation of the two detectors. By using two detectors in this fashion the scattering pattern produced by the average local structure may be mapped out by systematically changing the detector positions and cross correlating their outputs. Obviously this technique may be generalized in several ways by using more than one probe beam and more than two detectors. However, we limit our further discussions to this simpler two detector case.

We expect the cross correlation signal to be the strongest when the scattering volume and fluid correlation volumes are comparable. If the scattering volume is larger than the fluid correlation volume, then there will be interference between two or more fluid correlation regions which will reduce the nonguassian signal. (The nongaussian CCIFS signal is negligibly small compared to the Gaussian terms in the large scattering volume limit). If the scattering volume is less than the fluid correlation volume, the scattering pattern of the local structure will be diffraction broadened by the small illumination volume beyond that due to the finite size of the correlation volume. The broadened scattered intensity peaks will reduce any cross correlation signal. Thus we expect that the fluid correlation volume can be estimated by maximizing the cross correlation signal.

The average scattered intensity at a given momentum transfer or scattered wavevector $\bar{k}$ is sometimes viewed as a measure of the amplitude of a given periodic density fluctuation in the sample. This density fluctuation acts like a diffraction grating and produces a strong scattered signal at the corresponding $\bar{k}$ value in proportion to the grating amplitude. In a similar fashion, we may view the two detector cross correlation experiments as measuring the simultaneous existence of two periodic density fluctuations which (in general) have different wavelengths and orientations. The signal is strongest when the wavelength and orientation of these density waves corresponds to the probable orientation and structure in the sample. For large scattering volumes there is little orientational an translational order between different correlation volumes in fluids, and the cross correlation is negligibly small for density waves where the wave vectors are not identical. For small scattering volumes the local order exhibits correlation in density waves having different orientation, and the cross correlation signal can be significant.

\section{Experimental Demonstration}

The existence of a cross correlation spectrum has been demonstrated experimentally for a "two dimensional colloidal liquid". 13 . The sample is a monodisplerse aqueous suspension of $0.233 \mu \mathrm{m}$ diameter spheres which are charge stabilized and kept at minimal ionic strength by the presence of a strong acid strong base ion exchange resin. The sample volume contains a single monolayer of particles which are confined to the gap between the two quartz flats. Due to Coulomb interactions the particles repel one another and are repelled by surface 
$\mathrm{OH}^{-}$groups on the quartz flats. Because the quartz flats are not truly parallel but form a wedge, there is an electrostatic force on any given particle which pushes it towards larger gap spacing. Thus the sample density is observed to vary with the width of the gap. At very narrow gap spacing there are no colloidal particles, a "colloidal vacuum". As the gap spacing increases, the density continuously increases until a "colloidal fluid" state is evident. Further increase in the gap spacing $(\sim 10 \mu \mathrm{m})$ leads to a 2-dimensional hop "colloidal solid" with a well defined interface separating it from the fluid phase. This phase separation is similar to that observed in bulk samples in a gravitational field. The solid phase has a greater density than the fluid phase.

An argon-ion laser beam $(\lambda=4880 \AA)$ is directed normal to the plane of the sample and focussed to a $5.3 \mu \mathrm{m}$ diameter Gaussian beam waist as shown in figure 3. The scattered light may be projected onto a screen for viewing and video taping or fiber optic probes may be placed in the scattered field for cross correlation measurements. In the cross correlation experiments described here, the $k$-space radius of the position of both fiber optic probes $(|\bar{k}|$ and $|\bar{q}|)$ is equal to the $k$-space magnitude of the position of the first Debye Scherrer $\operatorname{ring}\left(|\vec{k}|=|\vec{q}|=k_{p S}\right)$. However, the two probes are separated by an azimuthal angle $\phi$ as measured with respect to the incient laser beam as an axis. Light collected by the $1 \mathrm{~mm}$ diameter fiber optic bundles is directed to separate photomultipliers and photon counting electronics. The resulting photopulse trains are fed to to a digital cross-correlator. Both zero delay time, equilibrium cross-correlation functions, or the time decay of cross-correlation functions can be studied.

Figure 2 presents "snap shots" of diffraction patterns produced by scattering from this sample. Figure $2 a$ shows the scattered intensity distribution $I(\bar{k})$ from the crystalline phase with the beam waist in the sample plane. The hexagonal close packed solid phase produces a diffraction pattern having six-fold symmetry with the low order $k$-space intensity maxima at $|\vec{k}|=k_{10}$. The lattice parameter is determined from $k_{10}$ to be $a=0.9$ um such that approximatiy 25 particles are being illuminated. Figure $2 \mathrm{~b}$ shows the intensity distribution when the beam waist is displaced from the sample plane such that the sample area illuminated has a larger diameter $(d=50 \mathrm{jm})$. In this fluid phase several correlation areas are being illuminated and the usual Debye scherrer ring of radius $|\bar{k}|=k_{D S}$ is observed. The speckles which form the ring have a typical dimension

$k_{10 H}-1 / d$. As the beam waist is moved into the sample plane, d decreases and becomes comparable to the fluid correlation length. The speckle size grows and becomes comparable to the width of the Debye Scherrer ring. When the two sizes are comparable, approximately one fluid correlation area is being illuminated at a given instant. Such regions exhibit local solid like order which is evident in the series of pictures in figure $2 \mathrm{c}-2 \mathrm{f}$. These patterns are not stationary, as in the solid phase, but flicker on and of changing in intensity and orientation every few tens of milliseconds. The k-space radius of the low order intensity maxima are the same as in the solid phase and also the same as the Debye Scherrer ring radius $\left(|\bar{k}|=k_{10}=k_{D S}\right)$. From this we conclude that the solid and the liquid have similar local SStructures and densities, however, the defects in the liquid phase give it a global disorder and lower global density.

Note that the scattering patterns all possess a reflection symmetry about an axis parallel to the plane of the sample and passing through the k-space origin. This is expected for scattering from a two dimensional sample in this geometry.

A typical cross correlation spectrum $\mathrm{C}(\phi, 0)$ is presented in figure 4 for scattering from the colloidal liquid phase near the colloidal solid phase boundary. The beam waist is in the sample plane such that the scattering area is comparable to the correlation area for the fluid. Because the instantaneous structure gives rise to the diffraction patterns presented in figure $2 c-f$, it is not suprising that this zero delay time cross correlation function exhibits strong 
positive peaks $(C(\phi, 0)>1)$ at $\phi=0,60,120,180^{\circ}$. There are anticorrelations between these peaks $(c(\phi, 0)<1)$. The amplitude of the peaks at $\phi=0$ and $180^{\circ}$ is larger than those at $\phi=60$ and $120^{\circ}$. This can be understond intuitively as shear distortions of the local structure which shift the positions of the scattered intensity peaks relative to one another. Such distortions will reduce the amplitude of cross correlated signals at $\phi=60$ and $120^{\circ}$ compared with the essentially autocorrelated peaks at $\phi=0$ and $180^{\circ}$.
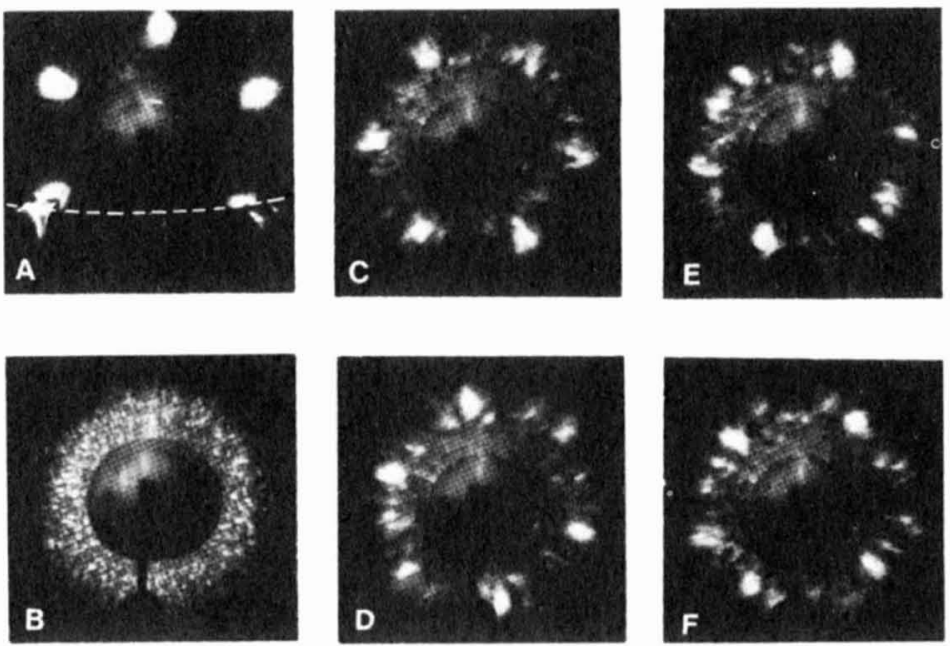

Fig. 2. Typically observed scattered light distributions obtained from video records of the scattered intensity on a screen parallel to the sample plane. A beam stop interrupts the incident laser beam at the centre of each photograph. (A) Scattering from the colloidal solid phase. The pattern is partially blocked by the cell edge, which is indicated by the dashed line.

(B) Scattering from the colloidal-iiquid phase using a large scattering area compared with fluid correlation area. (C)-(F) Scattering from the colloidalliquid phase using a scattering area comparable to the fluid correlation area. These pictures represent a time average over ca. $30 \mathrm{~ms}$. Note in $(C)-(F)$ the strong six-fold correlations and anticorrelations which are reminiscent of the solid phase. Note also in $(F)$ that $I(k)=I(-k)$,

The amplitude of the peaks at $\phi=0$ and $180^{\circ}$ are not the same, and this indicates that there may be some motion of the particles in a direction normal to the sample plane. Such motion destroys the two dimensional symmetry of the system and decreases the amplitude of the $\phi=180^{\circ}$ peak in this scattering geometry. 


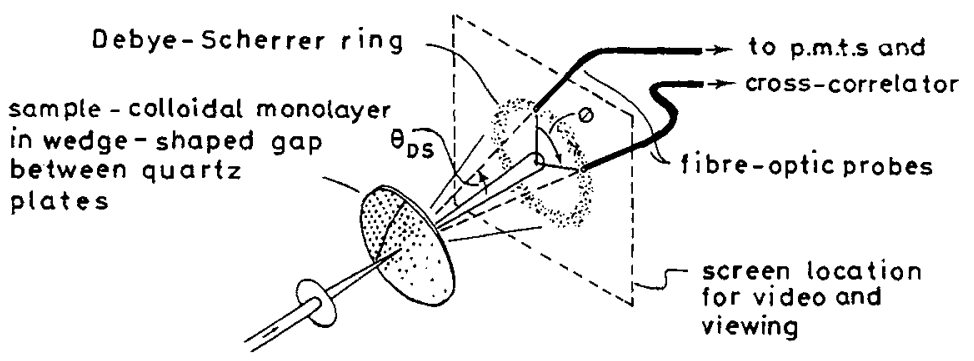

Figure 3. A laser light beam is directed normal to the plane of the sample. Measurements are made in the 'colloidal-fluid' phase away from the immediate vicinity of the liquid-crystal interface.

\section{Theoretical Considerations}

The normalized apertured-intensity cross correlation function in the Born scattering approximation is expressed as follows

$$
\begin{aligned}
& C(\bar{k}, \bar{q})=\langle I(\bar{k}) I(\bar{q})\rangle /\langle I(\bar{k})\rangle\langle I(\bar{q})\rangle \\
& =\int \varepsilon(\bar{r}) \varepsilon(\bar{r} \cdot) \varepsilon(\bar{r} \cdots) \varepsilon(\bar{r}+\cdots) \exp (i \bar{k} \cdot(\bar{r}-\bar{r} \cdot)+i \bar{q} \cdot(\bar{r} \cdots-\bar{r} \cdots)) \\
& \times P(\bar{r}, \bar{r} \cdot, \bar{r} \cdot 1, \bar{r}+\cdots) d \bar{r} d \bar{r} \cdot d \vec{r} \cdot d \bar{r}+\cdots / S(k) S(q)
\end{aligned}
$$

where the static structure factor is given by

$$
S(k)=\int E(\bar{r}) \varepsilon\left(\bar{r}^{\prime}\right) \exp \left(i \bar{k} \cdot\left(\bar{r}-\bar{r}^{\prime}\right)\right) P\left(\bar{r}, \bar{r}^{\prime}\right) d \bar{r} d \bar{r}^{\prime}
$$

Here the scattering volume is determined by the spatial extent of the field amplitude factor or aperture function, $E(\bar{r}) ; \bar{k}$ and $q$ are scattered wavevectors determined by the two detector positions relative to the incident beam, and the brackets enclosing the intensities $I(\bar{k})$ and $I(\bar{q})$ represent an equilibrium ensemble average over the multipoint number density $P$. We have assumed plane wavefronts are incident on the sample. For a beam waist coincident with the plane of a two dimensional sample, as in our experiments, this is a reasonable approximation. However, for a strongly focused beam in a three dimensional sample, the definition of $c(\bar{k}, \hat{q})$ presented in equation IV.1 needs further generalization.

The cross correlation function may be expressed in terms of particle correlation scattering functions up to fourth order as follows:

$$
\begin{aligned}
& C(\bar{k}, \bar{q})=\left[n ^ { 2 } \left\{\left[\rho_{2}(0)+n \rho_{11}(\bar{k},-\bar{k})\right]\right.\right. \\
& \times\left[\rho_{2}(0)+n \rho_{11}(\bar{q},-\bar{q})\right] \\
&+B\left[\rho_{2}(\bar{k}+\bar{q})+n \rho_{11}(\bar{k}, \bar{q})\right]\left[\rho_{2}(-\bar{k}-\bar{q})+n \rho_{11}(-\bar{k},-\bar{q})\right] \\
&\left.+B\left[\rho_{2}(\bar{k}-\bar{q})+n \rho_{11}(\bar{k},-\bar{q})\right]\left[\rho_{2}(\bar{q}-\bar{k})+n \rho_{11}(\bar{q},-\bar{k})\right]\right\} \\
&+\left[n \rho_{4}\left(\frac{0}{)}\right) \pm n^{2}\left[\rho_{31}(\bar{q},-\bar{q})+\rho_{31}(-\bar{q}, \bar{q})\right.\right. \\
&+p_{31}(\bar{k},-\bar{k})+p_{31}(-\bar{k}, \bar{k})+\rho_{22}(0,0)
\end{aligned}
$$




$$
\begin{aligned}
+ & \rho_{22}\left(\bar{k}+\bar{q},-\bar{k}-q \bar{q}+\rho_{22}(\bar{k}-\bar{q},-\bar{k}+\bar{q})\right] \\
+ & n^{3}\left[\rho_{211}(\overline{0}, \bar{q},-\bar{q})+\rho_{211}(\bar{k}+\bar{q},-\bar{k},-\bar{q})\right. \\
+ & \rho_{211}(-\bar{q}+\bar{k},-\bar{k}, \bar{q})+\rho_{211}(-\bar{k}+\bar{q}, \bar{k},-\bar{q}) \\
+ & \left.\rho_{211}(-\bar{q}-\bar{k}, \bar{k}, \bar{q})+\rho_{211}(0, \bar{k},-\bar{k})\right] \\
& \left.\left.+n^{4}\left[\rho_{1111}(\bar{k}, \bar{q})\right]\right\}\right] / s(\bar{k}) s(\bar{q})
\end{aligned}
$$

where the static structure factor is given by

$$
S(\bar{k})=n\left[\rho_{2}(0)+n \rho_{11}(\bar{k},-\bar{k})\right]
$$

and the apertured multiparticle scattering functions are defined by

$$
\begin{aligned}
& \rho_{n}(\bar{k})=\int d \bar{r} e^{i \bar{k} \cdot \bar{r}} \varepsilon^{n}(\bar{r}) f_{1}(\bar{r})
\end{aligned}
$$

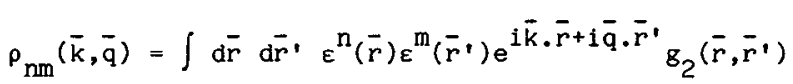

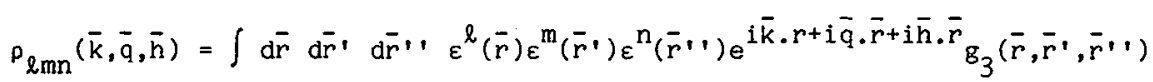

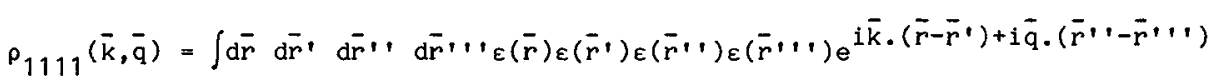

$$
\begin{aligned}
& \mathrm{g}_{4}\left(\overline{\mathrm{r}}, \overline{\mathrm{r}} \cdot, \overline{\mathrm{r}}^{\prime} \cdot, \overline{\mathrm{r}} \cdot \cdots\right)
\end{aligned}
$$

Here $\mathrm{n}$ is the average number density of the fluid and $\beta$ is a geometric signal to noise factor similar to that in dynamic light scattering. ${ }^{14}$ The single particle density is given by the $f_{1}(r)$ while $g_{n}\left(r_{1}, \ldots . r_{n}\right)$ are standard multiple particle correlation functions. ${ }^{15}$ The details of this derivation will be presented elsewhere. ${ }^{16}$ However, it is noteworthy to point out that this calculation assumes scattering from a homogeneous fluid and that the two detectors are positioned outside the diffraction broadened incident beam. Thus these results cannot be related directly to the small $|\bar{k}|,|\bar{q}|$ thermodynamic results of Knast and Chmielowski ${ }^{17}$.

The general structure of $\mathrm{C}(\phi, 0)$ has both Gaussian (first terms in curly brackets of equation 3) and nongaussian (second terms in curly brackets of equation 3) contributions. The Gaussian terms are important when $\bar{k}= \pm \bar{q}$ and become dominant for large scattering volumes. For large scattering volumes when $\bar{k}=\bar{q}$, we have the normal autocorrelation scattering geometry. ${ }^{7}$ When $\bar{k}=-\bar{q}$, we have ${ }^{a}$ scattering geometry which is used to reduce multiple scattering effects. 18. For small scattering volumes the nongaussian terms become more important. The $n \rho_{4}(0)$ term has been important in understanding number fluctuations ${ }^{19}$ and fluorescence photobleaching experiments. ${ }^{20}$ Kamm ${ }^{21}$ has pointed out the imporance of this term in determining the structure of anisotropic particles, while Griffith and Pusey ${ }^{22}$ and Kamm and Reich ${ }^{23}$ have measured its time decay to determine rotational diffusion constants. Pusey ${ }^{24}$ has argued the importance of the nongaussian single particle and pair distribution functions in determining the validity of the fundamental relation between number fluctuations and the pair correlation function. Finally, to describe the measured cross- 
correlation function presented in figure 4, the full expression given in equation 3 is required.

The expression for $\mathrm{C}(\phi, 0)$ presented in equation 3 is quite complex, and as a result we have examined simpler models to improve our intuition. Because the two dimensional colloidal liquid has a local solid-like structure, we have investigated the cross correlated scattering from a finite area of an infinite two dimensional harmonic solid. 25 For small displacements of particles which interact via central forces, the motion of particles in orthogonal direction is independent and the cross correlation function is unity at $\phi=90^{\circ}\left(\mathrm{C}\left(90^{\circ}, 0\right) \equiv 1\right)$. This is contrary to the experimentally observed result but is remedied theoretically by assuming that the reference lattice for the solid structure is randomly oriented on a time scale that does not interfere with lattice vibrations. This assumption also insures a uniform smearing out of the solid scattering pattern into Debye Scherrer rings for this model of the liquid phase. The results of this calculation are presented in figure 4 for a hexagonal array of seven particles in a uniformly illuminated scattering area with a nearest neightbor r.m.s. fluctuation of 0.2 times the lattice spacing. The results are for

$|\bar{k}|=|\bar{q}|=k_{n s}$ and are seen to be quite similar to the measured $c(\phi, 0)$. Evidently the thermally induced shear distortions of the solid phase have reduced the peaks at $\phi=60$ and $120^{\circ}$ compared to those at $\phi=0$ and $180^{\circ}$. This model for the liquid state is similar to the recent proposal by stillinger and Weber. 26 They suggest that the instantaneous fluid state is obtained by adding large lattice vibrations to a polycrystalline reference lattice, where the reference lattice becomes more polycrystalline as the temperature increases.

We have also begun investigations of the cross correlation function by a Monte Carlo simulation of a two dimensional collection of hard disks. Figure 4 presents the results of this calculation of $C(\phi, 0)$ for $|\bar{k}|=|\bar{q}|=k$ for a collection of 64 hard disks of which an average of thirteen are in the uniformly illuminated scattering volume at any given instant. The hard disk packing fraction (p.f. = .76) is near the freezing line for this system and correlations and anticorrelations are evident. Again the cross correlation function is quite similar to the experimentally measured function. Other results of this computer simulation will be reported elsewhere 16 and suggest a rather weak cross correlation effect until freezing is approached. The cross correlation may be stronger in systems which have longer ranged repulsive forces, as these forces will tend maintain constant particle separation and geometric order, as compared to the random filling of positional dead space in hard sphere systems.

\section{Discussion}

The method of cross correlation intensity fluctuation spectroscopy has been discussed in general and applied more speciflcally to two dimensional systems. The cross correlation spectrum for a two dimensional colloidal liquid has been obtained experimentally and has been compared with a two dimensional harmonic model and hard disk computer simulation. The results of these studies suggest the potential of this technique for the experimental, theoretical and computational investigation of the local order in fluid systems. Future efforts will explore several directions. The two dimensional studies should be extended to study $\mathrm{C}(\phi, 0)$ as a function of temperature or density as the freezing transition is approached. The cross correlation signal should increase as the fluid correlation length increases and the magnitude of the signal may serve as an order parameter for the fluid-solid transition. The time decay of $C(\phi, \tau)$ should also be investigated and is expected to show slow components as freezing is approached, due to the slow reorientation time for large clusters. Furthermore, it has been possible to predict the initial decay or first cumulant of $c(\phi, t)$ for "colloidal liquids". 27 Because these calculations reduce to equilibrium ensemble averages, 
Monte Carlo computer simulations can be used to predict initial time decays (and preliminary results indicate remarkable agreement with experimental measurements). The decay of orientational order in two dimensional melting may be studied by a generalization of this technique in which the local orientational order of two distinct scattering volumes are cross correlated and measured as a function of scattering volume separation. Studies should be initiated to look for icosohedral clusters in dense three dimensional fluids and glassy phases. Here the differences in $\mathrm{C}(\phi, 0)$ for $\mathrm{fcc}$, hcp and icosohedral structures may be estimated by using the coordinates of a finite cluster of particles with these symmetries in the formalism of Heer. 28 Finally the cross correlation technique may find application to pure atomic and molecular fluid systems as the requisite $x$-ray focussing technology becomes available.

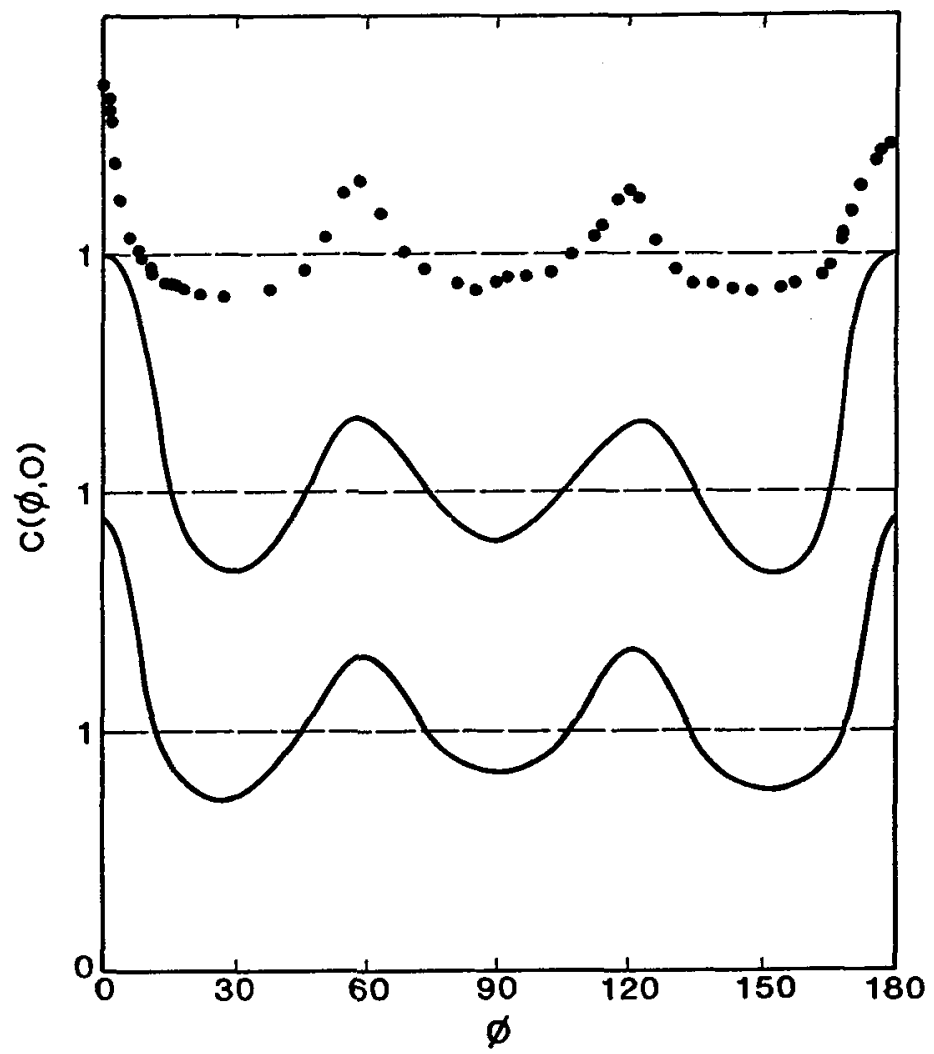

Figure 4. Upper curve: Experimentally measured values of the zero-time intensity cross-correlation function $C(\phi, 0)$ obtained from the two-dimensional colloidal liquid. Middle curve: Theoretical $\mathrm{C}(\phi, 0)$ function obtained for a rotationally averaged harmonic lattice model where the r.m.s. particle deviation of nearest neighbors is 0.2 times the lattice constant. Lower curve: Numerical calculation of $C(\phi, 0)$ for a Monte Carlo simultion of a hard disk fluid with a packing fraction of 0.76 .

The cross correlation technique is not without its problems. Care must be 
taken to have a well defined scattering volume with a known wavefront structure. Because the scattering volume is small, number fluctuations are a potential problem. The representation of $c(\phi, 0)$ in terms of particle correlation functions is very complex involving scattering volume weighted fourier transforms of multiple particle correlation functions. The resulting functions are highly symmetric in $\bar{k}$ and $\bar{q}$ and make extraction of three and four body correlation function difficult, if not impossible. Because local cluster orientation has more degrees of freedom in three dimensions than in two, the cross correlation signal is expected to be much smaller in three dimensions and will require longer experimental run times. However, these studies should be attempted because there is a great potential to learn more about local structure in fluid systems.

VI. Acknowledgements

The authors gratefully acknowledge support by the National Science Foundation, Division of Materials Research, Low Temperature Physics Program grant numbers DMR 82-06472 for N.A.C. and DMR 81-16119 for B.J.A.

VII. References

1. B. E. Warren, X-ray Diffraction (Addison-Wesley Publishing Company, Reading, 1969).

2. C. Tanford, Physical Chemistry of Macromolecules (John Wiley, New York, 1961).

3. N. A. Clark, A. J. Hurd and B. J. Ackerson, Nature (London) 28157 (1979).

4. A. J. Hurd, N. A. Clark, R. C. Mockler and W. J. O'Sullivan, Phys. Rev. A26 2869 (1982).

5. P. Pieranski, E. Dubois-Voilette, F. Rothen and L. Strzelecki, J. Phys. (Paris) $4253(1981)$.

6. S. H. Chen, B. Chu and R. Nossal, Scattering Techniques Applied to Supramolecular and Nonequilibrium Systems (Plenum Press, New York, 1981 ).

7. B. J. Berne and R. Pecora, Dynamic Light Scattering (John Wiley, New York, 1976).

8. P. A. Egelstaff, An Introduction to the Liquid State (Academic Press, London, 1967).

9. J. A. Prins, Naturwissenshaften, 19435 (1931); J. A. Prins and H. Peterson, Physica, 3147 (1931).

10. P. J. Steinhardt, D. R. Nelson and M. Ronchetti, Phys. Rev. B28 784 (1983).

11. F. C. Frank, Proc. Roy. Soc. Lond. Ser. A215 43 (1952).

12. B. J. Halperin and D. R. Nelson, Phys. Rev. Lett: 41121 (1981); D. R. Nelson and B. J. Halperin, Phys. Rev. B19 2457 (1979).

13. N. A. Clark, B. J. Ackerson and A. J. Hurd, Phys. Rev. Lett., 501459 (1983).

14. V. Degiorgio and J. B. Lastovka, Phys. Rev. A4 2033 (1971).

15. Y. L. Klimontovich, Statistical Theory of Nonequilibrium Processes in a Plasma (M.I.T. Press, Cambridge, 1967).

16. B. J. Ackerson, T. W. Taylor and N. A. Clark (to be published).

17. K. Knast and W. Chmielowski, J. Physique 421373 (1981).

18. G. D. J. Phillies, J. Chem. Phys. $74260 \overline{(1981) ; ~ P h y s . ~ R e v . ~ A 24 ~} 1939$ (1981).

19. D. W. Schafer and B. J. Berne, Phys. Rev. Lett. 28475 (1972).

20. D. Madge, Q. Rev. Biophys. 935 (1976).

21. Z. Kam, Biophys. J. 397 (1982); Macromolecules 10922 (1977).

22. W. G. Griffin and P. N. Pusey, Phys. Rev. Lett. $\frac{43}{1100}$ (1979).

23. S. Reich and Z. Kam; Opt. Commun. 30293 (1979).

24. P. N. Pusey, J: Phys. A12 1805 (1979).

25. B. J. Ackerson and N. $\overline{A .}$ Clark, Faraday Discussions Chem. Soc. 76219 (1983).

26. F. H. Stillinger and T. A. Weber, Phys. Rev. A25 978 (1982).

27. R. J. A. Tough, P. N. Pusey and B. J. Ackerson, (to be published in J. Chem. Phys.).

28. C. V. Heer, Optics Comm. 38110 (1981). 